\title{
Empreendedorismo eletrônico: o uso da Educação Corporativa na formação de empreendedores
}

\author{
Arcângelo dos Santos Safanelli - UFSC (PPGAU) - arcangelosafanelli@ hotmail.com \\ Bruno César de Melo Moreira - UFSC (CPGA) - bcmmoreira @ yahoo.com.br
}

Resumo: Nas últimas décadas viu-se o desenvolvimento avassalador de tecnologias que permitem, cada vez mais, interconectar o indivíduo a um mundo novo e repleto de possibilidades. Uma amostra disto é o crescente comércio através da web, que por um lado apresenta novas oportunidades, e por outro, uma carência de novos e específicos conhecimentos. Neste cenário surge o empreendedorismo eletrônico. Aliando técnicas convencionais às originadas exclusivamente para 0 mercado on-line, 0 empreendedorismo eletrônico, formulado e oferecido como prática de Educação Corporativa visa promover o desenvolvimento de habilidades específicas do empreendedorismo no contexto da web. Desta forma, o objetivo do presente trabalho é discutir como a Educação a Corporativa possibilita a disseminação de novos conhecimentos, como os envolvendo o ensino da cultura empreendedora, requeridos neste contexto de globalização. Para tanto, será apresentado e discutido o caso da EMPREEND/UnB - Escola de Empreendedores da Universidade de Brasília. Nota-se, portanto, que a Educação Corporativa tem evoluído e se tornando uma realidade no país, mesmo em grandes universidades, contribuindo para a disseminação, formação e estímulo a pesquisas, em temas específicos, que forneçam respostas ao mercado na velocidade que ele necessita a fim de gerar inovações e competitividade para as empresas.

Palavras Chaves: Educação a Distância, Empreendedorismo, Inovação.

\section{Eletronic entrepreneurship: the use of Corporate Education in the training of entrepreneurs in the e-commerce era}

\begin{abstract}
In the last decades have seen the overwhelming development of technologies which allow increasingly interconnect the individual to a new world full of possibilities. A sample of this is the growing trade across the web, which on one hand presents new opportunities, and secondly, a lack of new and specific knowledge. In this context arises the e-entrepreneurship. By combining conventional techniques to originate exclusively for the online market, entrepreneurship, electronics, formulated and offered as a practice of Corporate Education aims to develop specific skills of entrepreneurship in the context of the web. Thus, the aim of this paper is to discuss how Corporate Education allows dissemination of new knowledge, such as those involving the teaching of entrepreneurial culture, required in this context of globalization. For this purpose, will be presented and discussed the case to Project / UnB - School of Entrepreneurship at the University of Brasilia. Note, therefore, that the corporate education has evolved and is becoming a reality in the country, even in large universities, contributing to the dissemination, training and encouragement of research on specific topics, providing
\end{abstract}


answers to the market at the speed it needs in order to generate innovation and competitiveness for companies.

Keywords: Distance Education, Entrepreneurship, Innovation. 


\section{INTRODUÇÃO}

Nas últimas décadas viu-se o desenvolvimento avassalador de tecnologias que permitem, cada vez mais, interconectar o indivíduo a um mundo novo e repleto de possibilidades. Os avanços recentes nas áreas da computação, telecomunicações e tecnologias da informação, estão mudando a maneira de se ver o mundo. Dentro do cenário econômico, com a internet, novos horizontes surgem. Uma amostra disto é o crescente comércio através da web.

A própria Organização Mundial do Comércio - OMC (1998) ressalta:

These modern technologies are being combined, especially through the Internet, to link millions of people in every corner of the world. Communications are increasingly unburdened from the constraints of geography and time. Information spreads more widely and more rapidly than ever before. Deals are struck, transactions completed, and decisions taken in a time-frame that would have seemed simply inconceivable a few years ago. This technological revolution will increasingly touch every area of activity where the digital transmission of information serves a purpose, whether it be in the office, in business, or in the worlds of shopping, leisure and entertainment. (OMC, 1998, p.1).

Na mesma direção argumenta Tigre (1999) sobre o papel da internet neste novo contexto de globalização:

A internet constitui uma poderosa ferramenta para facilitar e multiplicar a comunicação global entre pessoas e instituições. Do ponto de vista econômico, seu potencial é refletido principalmente através do comércio eletrônico, uma aplicação das tecnologias da informação direcionada para apoiar processos produtivos e transações de bens e serviços. O chamado ecommerce permite fortalecer a rede global de produção, comércio e tecnologia e os vínculos internos das corporações em uma ampla gama de situações. (Tigre, 1999, p.85).

A evolução do comércio eletrônico ou e-commerce, motivada pelo desenvolvimento de novas tecnologias da informação e comunicação, proporciona, por um lado, novas alternativas e oportunidades, e por outro, uma carência de novos e específicos conhecimentos e saberes.

Cada vez mais se torna complexa a tarefa de iniciar e manter um novo empreendimento. Novas competências são, portanto, criadas e exigidas para que as empresas se originem e se mantenham ativas neste cenário que se apresenta. Dentro deste paradigma globalizado as empresas mais que tudo precisam inovar.

Buscando minimizar este problema novas abordagens educacionais ganham destaque. Aliando ferramentas tecnológicas e conhecimentos baseados nas novas necessidades, a Educação Corporativa apresenta-se como uma modalidade de ensino que busca oferecer orientações estratégicas alinhada à esta necessidade de qualificação profissional. Quando se refere especificamente aos novos conhecimentos exigidos por 
este imenso e competitivo mercado on-line, a Educação Corporativa pode contribuir ainda de maneira mais decisiva. Neste cenário surge o empreendedorismo eletrônico.

Assim, aliando técnicas convencionais às originadas exclusivamente para o mercado online, o empreendedorismo eletrônico, formulado e oferecido como prática de Educação Corporativa se mostra uma forma de educação que visa promover o desenvolvimento de habilidades específicas do empreendedorismo em um contexto web.

Neste contexto, o objetivo do presente trabalho é discutir como a Educação a Corporativa possibilita a disseminação de novos conhecimentos, requeridos neste contexto de globalização, apresentando para tanto, uma experiência de ensino on-line envolvendo o ensino da cultura empreendedora.

A intenção é apresentar e suscitar uma discussão a respeito desta problemática. Muitos conhecimentos requeridos neste contexto de globalização podem ser oferecidos virtualmente, utilizando-se de técnicas e ferramentas adequadas, proporcionando uma melhoria na qualificação da mão-de-obra existente, resultando em um ganho de competitividade e gerando desenvolvimento ao país.

\section{O EMPREENDEDORISMO NO CONTEXTO DA EDUCAÇÃO CORPORATIVA}

Segundo Oliveira Filho (2010), empreendedorismo é um processo ou uma cadeia de eventos que modificam de forma inovadora as atividades de organização, administração, execução, geração de riquezas, transformação de conhecimentos e bens em novos produtos dentro de uma organização. Para o autor:

Empreendedor é o termo utilizado para qualificar, ou especificar, principalmente, aquele indivíduo que possui uma forma especial, inovadora, de se dedicar às atividades de organização, administração, execução; principalmente na geração de riquezas, na transformação de conhecimentos e bens em novos produtos - mercadorias ou serviços; gerando um novo método com o seu próprio conhecimento. É o profissional inovador que modifica, com sua forma de agir, qualquer área do conhecimento humano. (Oliveira Filho, 2010, p.11).

Já conforme Britto e Wever (2003, p. 17), "uma das primeiras definições da palavra empreendedor, foi elaborada no início do século XIX pelo economista francês J. B. Say, como aquele que transfere recursos econômicos de um setor de produtividade mais baixa para um setor de produtividade mais elevada e de maior rendimento"

Contudo, parece que uma definição de empreendedor que atende na atualidade é de Dornelas (2001, p. 37), que está baseada nas diversas definições vistas até então,"o empreendedor é aquele que detecta uma oportunidade e cria um negócio para capitalizar sobre ela, assumindo riscos calculados".

Segundo Dornelas (2001), o empreendedorismo ganhou força no Brasil somente a partir da década 1990, com a abertura da economia que propiciou a criação de entidades como SEBRAE (Serviço Brasileiro de Apoio às Micro e Pequenas Empresas) e SOFTEX (Sociedade Brasileira para Exportação de Software).

Drucker (1986) entende que empreendedor não é só aquele que se envolve em atividades econômicas, mas a pessoa que assume riscos calculados e planejados sabe lidar com as incertezas, possui métodos de trabalho claros e organizados e busca 
trabalhar e conviver com pessoas que façam sempre o melhor de forma diferenciada e inovadora. Assim, uma pessoa que busca se capacitar e aprender por meio da da Educação Corporativa pode ser considerada empreendedora. De acordo com pesquisas realizadas pelo Serviço Brasileiro de Apoio a Micro e Pequenas Empresas - SEBRAE (2001), são dez as principais características do comportamento empreendedor e é com base em algumas das:

1. Estabelecimento de metas claras e definidas;

2. Busca constante por oportunidades e habilidade para identificá-las;

3. Capacidade de assumir riscos calculados;

4. Busca por informações, inovação e conhecimento do ramo em que empreende;

5. Planejamento e monitoramento sistemático do projeto;

6. Exigência de qualidade e eficiência com senso apurado de organização.

7. Persistência, otimismo e flexibilidade;

8. Comprometimento com a empresa e com seus colaboradores;

9. Grande capacidade de persuasão e formação de extensa rede de contatos;

10. Independência e autoconfiança com disposição para tomar decisões e liderar equipes.

A busca constante por informações e conhecimento do ramo em que se encontra também é uma forte característica empreendedora. Drucker (1986) lembra que o empreendedor está sempre atento às mudanças (pretensas ou efetivas) de um determinado setor ou do mercado e, a sua percepção aguçada para essas reconfigurações que demandam atitudes rápidas com vistas à soluções inovadoras e criativas estão fortemente ligadas à sua atualização e conhecimento de suas atividades.

Neste contexto, como forma que mediatiza a ação educativa, acredita-se que o principal desafio da Educação Corporativa no mundo contemporâneo é dar conta das intensas e freqüentes demandas dos mercados que exigem sempre mecanismos inovadores para a qualificação de seus profissionais. Nesse sentido, é possível identificar uma característica de atitude empreendedora, a busca pela inovação.

Para Moore (2003, p. 56), "esse processo de transformação que vem acontecendo no modelo educacional é chamado de mudança organizacional, já que há uma sensível alteração na dinâmica interna da cultura institucional”.

Entretanto, como argumenta Kollmann (2006), em tempos recentes o avanço das tecnologias de informação dentro das empresas e mercados, tem possibilitado o desenvolvimento de conceitos de negócios inovadores, baseados em informações eletrônicas e redes de comunicação. Como salienta o autor, neste contexto nasce o termo Empreendedorismo Eletrônico, que se caracteriza pelo processo de criação de empresas específicas para este mundo digital.

Para o autor, estabelecer uma empresa dentro deste mundo configurado pelas novas tecnologias de informação e comunicação, requer conhecimentos específicos sobre fatores correlatos à este novo tipo de economia. Neste sentido, novas práticas devem ser adotadas visando inserir ao contexto da organização aspectos específicos do e-commerce. Nasce uma necessidade crescente de se capacitar os indivíduos, de 
maneira rápida, prática e que possibilite assimilar conhecimentos específicos voltados para esta nova área que surge.

Na seção 3 apresentar-se-á a Educação Corporativa como sucesso da experiência da EMPREED/CDT/UNB no ensino de conhecimentos específicos relacionados à cultura empreendedora.

\section{3. - EDUCAÇÃO CORPORATIVA E A EXPERIÊNIA DA EMPREEND/CDT/UNB}

Neste contexto de novos conhecimentos, de uma economia dinâmica, as empresas estão buscando sempre capacitar seus profissionais para enfrentar os novos desafios. Segundo Eboli, Hourneaux Jr. e Mancini (2005),

$\mathrm{Na}$ sua incessante busca pela perpetuidade do negócio e aumento de competitividade no mercado, muitas empresas têm buscado novas formas para o aprendizado e desenvolvimento dos seus trabalhadores e, muitas vezes, também de seus públicos interessados externos. Como uma alternativa para incrementar a capacitação individual e, conseqüentemente, gerar níveis mais altos de competência para toda a organização, surgiu o conceito de Educação Corporativa, que se consolidou na década de 1990 nos Estados Unidos e que vem ganhando cada vez mais espaço no Brasil. (Eboli, Hourneaux Jr. e Mancini, 2005, p.1).

Uma importante ferramenta para tanto, é a Educação Corporativa. A educação corporativa vem apresentar um modelo de educação em consonância com este novo mundo, plural e globalizado em que nos encontramos.

De acordo com o Ministério do Desenvolvimento, Indústria e Comércio Exterior (2011):

Educação Corporativa pode ser definida como uma prática coordenada de gestão de pessoas e de gestão do conhecimento tendo como orientação a estratégia de longo prazo de uma organização. Educação corporativa é mais do que treinamento empresarial ou qualificação de mão-de-obra. Trata-se de articular coerentemente as competências individuais $\mathrm{e}$ organizacionais no contexto mais amplo da empresa. Nesse sentido, práticas de educação corporativa estão intrinsecamente relacionadas ao processo de inovação nas empresas e ao aumento da competitividade de seus produtos (bens ou serviços).

Neste cenário de constantes inovações tecnológicas, Martins e Fuerth (2011) ressaltam que a Educação Corporativa se mostra como uma importante ferramenta na busca por vantagem competitiva.

A globalização afeta todas as áreas da sociedade, todas as mudanças giram em torno da tecnologia de informação. O 
mundo virtual não pára de crescer. Dentro do nosso atual contexto profissional, muito já se ouviu falar de treinamento e desenvolvimento, aprendizagem organizacional e gestão do conhecimento. Atualmente estamos vivenciando uma metamorfose educacional, ou seja, a substituição do antigo Treinamento e Desenvolvimento (T\&D) pela educação corporativa. (Martins e Fuerth, 2011, p.2).

Para os autores, a Educação Corporativa "Trata-se de um modelo estruturado utilizado para transmitir conhecimentos específicos sobre determinados assuntos dos quais os funcionários possam estar apresentando alguma deficiência, e também para prepará-los para os desafios vindouros". (Martins e Fuerth, 2011, p.14).

Um dos melhores exemplos de Educação Corporativa voltada para o ensino de um conhecimento específico, o empreendedorismo, é apresentado pela EMPREEND Escola de Empreendedores.

A EMPREEND é um dos vários programas do Centro de Apoio ao Desenvolvimento Tecnológico da Universidade de Brasília (CDT/UnB), e tem seu foco de atuação voltado principalmente para universitários e empreendedores. Nesse sentido, tem realizado diversas atividades de forma a incentivá-los e a capacitá-los para a atividade empreendedora.

Desde 1996, a EMPREEND/CDT oferece disciplinas voltadas à disseminação da cultura empreendedora para diversas áreas do conhecimento em nível de graduação na UnB, além de cursos de extensão e de especialização com vistas à formação de empreendedores. Dentre os seus objetivos, podem ser elencados aqueles voltados para a capacitação, formação e estímulo a estudos e pesquisas que forneçam respostas ao mercado na velocidade que ele necessita (e demanda) a fim de gerar inovações e competitividade para as empresas. Uma das conseqüências desse trabalho é a disponibilização de informações estratégicas que agregam valor competitivo às empresas e aos seus negócios.

Assim, o objetivo principal dos cursos oferecidos pela EMPREEND/CDT/UnB, é capacitar gestores de telecentros para garantirem a sustentabilidade dos telecentros a partir dos temas abordados nos cursos "Gestão de Telecentros" e "Empreendedorismo Digital". Os cursos promoveram uma interação com a comunidade divulgando as ações promovidas nos "blogs" do telecentros para que a comunidade tomasse conhecimento. Neste sentido, busca-se estimular e promover a capacidade do aluno pensar de forma crítica, criativa, responsável e inovadora.

Os cursos de extensão para capacitação de gestores de Telecentros de Informação e Negócios foram pesquisados e desenvolvidos por meio de novos paradigmas de comunicação, resultantes do desenvolvimento de plataformas tecnológicas na concepção de ambientes de aprendizagem interativos para cursos a distância, voltados para o ensino e aprendizagem organizacional.

Destaca-se a realização dos estudos e pesquisas que viabilizaram o desenvolvimento editorial, feitos no período de janeiro a março de 2007, por uma equipe multidisciplinar de pesquisadores e especialistas em tecnologias educativas, gestão do conhecimento e objetos de aprendizagem para uma análise situacional dos TIN's e visão de negócios. Durante o desenvolvimento foram analisadas as necessidades de conteúdo, ambiente virtual, referências bibliográficas, revisão gramatical e o projeto gráfico, dentre outros, o que possibilitou a identificação da metodologia para a organização dos conteúdos dos 10 cursos e sua tradução sob a forma 
de hipertextos. Após a conclusão da pesquisa do desenvolvimento do projeto gráfico, pedagógico e implementações educativas, foi apresentada a proposta final de capacitação dos gestores TIN’s, que foi dividida em duas etapas (MDIC, 2010, p. 16):

1. Capacitação Gerencial - dividida em 5 (cinco) módulos destinados aos gestores dos TIN's, para subsidiá-los em sua missão de gerenciamento dos telecentros e;

2. Capacitação Operacional - com 5 (cinco) módulos, destinados ao público em geral (gestores dos TIN'S, multiplicadores, empreendedores, comunidade), voltados para o desenvolvimento de habilidade e conhecimento para empreender.

Os cursos foram executados em duas etapas: Curso de Gestão de Telecentros realizado no período de março a outubro de 2007, e o Curso de Empreendedorismo Digital - realizado no período de agosto a outubro de 2007.

As capacitações foram destinadas a gestores de telecentros de informação e negócios, promovidas pelo Ministério do Desenvolvimento, Indústria e Comércio Exterior - MDIC, em parceria com o Centro de Apoio ao Desenvolvimento Tecnológico - CDT, da Universidade de Brasília - UnB.

A fim de minimizar os custos e deslocamentos dos participantes, os cursos foram realizados a distância, por meio do uso da Internet, contando com tutores que realizados a distância, por meio do uso da Internet, contando com tutores que estiveram disponíveis on-line, para acompanhamento pedagógico, orientação, perguntas, dúvidas e esclarecimentos.

Os cursos de capacitação foram desenvolvidos por especialistas de reconhecida atuação nas áreas de pesquisa, pedagogia, designer gráfico e Educação a Distância, que levaram em consideração os seguintes critérios (MDIC, 2010, p. 15):

- contexto e cenários atuais dos Telecentros de Informação e Negócios;

- clareza de objetivos, coerência e consistência do projeto;

- compatibilidade da proposta com o público-alvo;

- conteúdo e metodologia dos cursos;

- adequação e garantia da vivência prática;

- potencial de desenvolvimento do empreendedorismo;

- viabilidade do cronograma de desenvolvimento do projeto

A carga horária foi de 300 horas de duração, distribuídas em duas etapas de 5 módulos, sendo que cada módulo totalizou 30 horas/aula de curso. De forma a ressaltar a importância da Educação Corporativa e do potencial tecnológico passível de ser por ela utilizado - tanto no contexto acadêmico quanto no contexto das organizações de trabalho -, são algumas das benesses promovidas por essa forma de ensino a transformação da estrutura e mudanças nos ambientes educacionais (onde o indivíduo possa estudar de forma autônoma, de forma a conduzir seu processo criativo com interatividade, criatividade e flexibilidade).

Agilidade nas trocas de informações com ênfase na metacognição, a elaboração de currículos mais flexíveis, a motivação para estimular o desenvolvimento do pensar crítico e criativo, a preocupação com questões sociais, a valorização e a diversificação 
da educação, a ampliação das oportunidades de aprendizagem, entre outros. Guardando as devidas e necessárias proporções, verificam-se aqui parte das características empreendedoras apresentadas no início deste trabalho. (Gomes; Pimenta, 2010).

A experiência da EMPREEND/CDT/UnB tem demonstrado o resultado satisfatório do programa, na formação de empreendedores através dessa forma de tecnologia.

\section{CONCLUSÕES E REFLEXÕES}

O presente trabalho teve como intuito incitar o debate sobre um ponto crucial dentro deste novo paradigma de educação que se abre no Brasil e no mundo que é a Educação Corporativa disseminando conhecimentos específicos nesta nova realidade globalizada, dinâmica e competitiva que nos cerca.

Em um mundo marcado pela inovação tecnológica, pela competitividade, pelo dinamismo, cada vez mais as empresas precisam estar preparadas e atualizadas dentro do novo conhecimento. Em relação ao empreendedorismo não é diferente. Sendo um processo que modifica de forma inovadora as atividades de organização, administração, execução, geração de riquezas, transformação de conhecimentos e bens em novos produtos dentro de uma organização, este se mostra um tipo de conhecimento cada vez mais demandado.

Assim, as empresas estão buscando capacitar seus profissionais para enfrentar estes novos desafios. Neste contexto, como demonstrado na seção 3, nasce a Educação Corporativa. Esta se mostrou como um modelo de educação estruturado, em consonância com este novo mundo, plural e globalizado em que nos encontramos, buscando transmitir conhecimentos específicos sobre determinados assuntos, garantindo capacitação, formação e estímulo a estudos e pesquisas que forneçam respostas ao mercado na velocidade que ele necessita (e demanda) a fim de gerar inovações e competitividade para as empresas.

Como caso de sucesso da Educação Corporativa no ensino de conhecimentos específicos relacionados à cultura empreendedora, foi apresentado, portanto, a experiência da EMPREED/CDT/UNB.

Criado por uma equipe multidisciplinar de pesquisadores e especialistas em tecnologias educativas, gestão do conhecimento e objetos de aprendizagem, os métodos empregados pela EMPREED/CDT/UNB, como forma de Educação Corporativa, garantem uma agilidade nas trocas de informações, a elaboração de currículos mais flexíveis, a motivação para estimular o desenvolvimento do pensar crítico e criativo, a preocupação com questões sociais, a valorização e a diversificação da educação, a ampliação das oportunidades de aprendizagem, entre outros, conferindo à proposta de ensino, elementos essenciais para o seu sucesso.

Conforme foi possível observar, as competências empreendedoras elencadas e analisadas são passíveis de serem alinhadas com as principais características dos alunos que obtém sucesso nos estudos a que se dedicam e do empreendedor bem-sucedido.

Nota-se, portanto, que a Educação Corporativa tem evoluído e se tornando uma realidade no país, mesmo em grandes universidades, contribuindo para a disseminação, formação e estímulo a pesquisas, em temas específicos, que forneçam respostas ao mercado na velocidade que ele necessita a fim de gerar inovações e competitividade para as empresas. 


\section{REFERÊNCIAS BIBLIOGRÁFICAS}

BRITTO, Francisco; WEVER, Luiz. Empreendedores brasileiros: vivendo e aprendendo com grandes nomes. Rio de Janeiro: Campus, 2003.

DORNELAS, José Carlos Assis. Empreendedorismo: transformando idéias em negócios. Rio de Janeiro: Elsevier, 2001.

DRUCKER, Peter. Inovação e espírito empreendedor: Prática e princípios. São Paulo: Pioneira, 1985.

EBOLI, M.; HOURNEAUX JR, F.; MANCINI, S. Breve Panorama da Educação Corporativa no Brasil: Apresentação de Resultados de Pesquisa. In: XXIX ENCONTRO DA ASSOCIAÇÃO NACIONAL DOS PROGRAMAS DE PÓSGRADUAÇÃO EM ADMINISTRAÇÃO, 2005, Brasília, Anais. Brasília: Programas de Pós- graduação em Administração.

GOMES, M. F. O.; PIMENTA, F. F. P. EaD Empreendedora: o uso da tecnologia como fator de sucesso na Educação a Distância nos cursos de formação e treinamento de profissionais - A primeira EMPREEND/CDT/UnB. Disponível em: <www.cdt.unb.br/pdf/EAD_Artigo_revista_participacao.pdf>. Acesso em dezembro de 2010 .

KOLLMANN, T. What is e-entrepreneurship? Fundamentals of company founding in the net economy. International Journal of Technology management, v.33, n. 4. 2006.

MARTINS, A.; FUERTH, L. R. A educação corporativa e o processo de requalificação profissional das empresas brasileiras. Disponível em: <http://www.lilianramos.net/downloads/>. Acesso em: 12 de maio de 2011.

MINISTÉRIO DO DESENVOLVIMENTO, INDÚSTRIA E COMÉRCIO EXTERIOR. Telecentros. Disponível em: <http://www.telecentros.desenvolvimento.gov.br/sitio/inicial/index.php>. Acesso em Abril de 2010.

MINISTÉRIO DO DESENVOLVIMENTO, INDÚSTRIA E COMÉRCIO EXTERIOR. Portal de Educação Corporativa. Disponível em: <http://www.educor.desenvolvimento.gov.br/educacao>. Acesso em: 22 de março de 2011.

MOORE, M.; KEARSLEY, G. Distance Education: a systems view. United State: Wadsworth Publishing Company, 2003.

OLIVEIRA FILHO, J. B. Empreendedorismo. Florianópolis: Universidade Federal de Santa Catarina / Sistema UAB. 2010. 150 p. 
TIGRE, P. B. Comércio eletrônico e globalização: um desafio para o Brasil. In: LASTRES, H.; ALBAGLI, S. (Ed.) Informação e Globalização na Era do Conhecimento, Comércio Eletrônico e Globalização: Desafios para o Brasil. Rio de Janeiro: Editora Campus, 1999. 